\title{
Capacity of the Energy Harvesting Channel with Energy Arrival Information at the Receiver
}

\author{
Omur Ozel $^{1}$, Kaya Tutuncuoglu ${ }^{2}$, Sennur Ulukus ${ }^{1}$, and Aylin Yener ${ }^{2}$ \\ ${ }^{1}$ Department of Electrical and Computer Engineering, University of Maryland, College Park, MD 20742 \\ ${ }^{2}$ Department of Electrical Engineering, The Pennsylvania State University, University Park, PA 16802
}

\begin{abstract}
We determine the capacity of a discrete memoryless communication channel with an energy harvesting transmitter and the energy arrival information available at the receiver as well as the transmitter. We obtain an $n$-letter capacity expression and prove that the capacity is achieved by an encoding scheme that depends only on the current battery state. Moreover, the capacity is invariant to the non-causal knowledge of energy arrivals. Finally, we show that the capacity expression is equivalently the maximum directed mutual information and that the channel output feedback does not increase the capacity in this case. We obtain upper and lower bounds on the capacity and numerically evaluate them for comparison.
\end{abstract}

\section{INTRODUCTION}

In this paper, we study a communication channel with an energy harvesting transmitter where energy arrival information is available at the receiver in addition to the transmitter as shown in Fig. 1. In this channel, the energy of each transmitted code symbol is constrained to the available battery energy in that channel use. The exogenous energy arrival process replenishing the battery energy at the transmitter is independent of the message. The capacity of this channel with only transmitter side information has been determined in recent work for two extreme cases in the Gaussian setting: When the battery size is unlimited, [1] showed that the capacity is equal to the capacity of the same system with an average power constraint equal to the average recharge rate. When the battery size is zero, [2] showed that the capacity is achieved by using Shannon strategies [3]. Moreover, the recent work [4] explored the capacity of the energy harvesting channel with battery state information at both sides. In this paper, we complement the results in [4] by determining the capacity of the same system when energy arrival information is available at both sides.

The energy harvesting channel is a state-dependent channel where state process has memory and is input dependent. We follow previous work in [4]-[7] and model the energy arrivals as multiples of a fixed quanta. Consequently, we obtain a physical layer which has a discrete alphabet based on this quanta. Unlike the case of battery state information at the receiver side [4], resulting channel is not a Markov channel when energy arrival side information is available at the receiver. In addition, the state information of this nonMarkov channel is available at both the transmitter and the receiver.

This work was supported by NSF Grants CNS 09-64632/CNS 09-64364, and CCF 14-22111/CCF 14-22347.

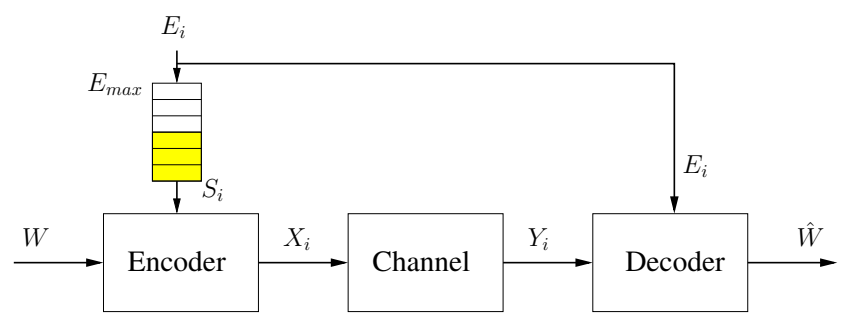

Fig. 1. The channel with an energy harvesting transmitter with a finite-sized battery. The energy arrival information is available at both sides.

In this paper, we determine the capacity of this channel as the limit of an $n$-letter maximum information rate. This expression reveals two crucial characteristics regarding the best achievable rate when energy arrival information is made available to the receiver: It suffices to use only current battery energy level in the encoding to achieve capacity. Reference [7] conjectures that for an energy harvesting channel with only the transmitter side energy arrival information, coding based only on the current battery energy level is optimal. Our results show that when both the transmitter and the receiver have the energy arrival information, coding based only on the current battery energy level is optimal. Secondly, noncausal knowledge of energy arrivals at the transmitter does not improve the best achievable rate. Our work also relates to the recent work [8], [9]. In particular, bounds for the capacity with and without energy arrival side information are studied in [8]. Moreover, when the energy arrivals are deterministic as in [9], the receiver automatically has the energy arrival information and hence our results apply to the setting in [9].

In energy harvesting communications, there are inherent energy causality constraints on the transmitted code symbols. These constraints have been well studied in the literature on transmission scheduling, see e.g., [10]-[12]. In our current paper, we explicitly use these causality constraints and resulting directionality of energy at the channel use level. We determine that the capacity expression is equivalently expressed as the maximum directed mutual information between the channel input and the channel output and energy. This enables us to determine the impact of feedback on the capacity of the energy harvesting channel. Note that available side information at the receiver side can be viewed as a delayed feedback. As the energy arrival and real channel output form an extended output, only partial output feedback is available in this case. 
We find that additional channel output feedback does not increase the capacity.

\section{The Channel Model}

We consider a channel with an energy harvesting transmitter. The battery in the transmitter can store at most $E_{\max }$ units of energy. Input symbols lie in the set $\{0,1, \ldots, K\}$ with the convention that each symbol $k$ has $k$-unit energy cost where $K \leq E_{\max }$. When channel input $X_{i}$ is transmitted in the $i$ th channel use, the receiver gets $Y_{i}$. Underlying physical channel is a DMC and is determined by the stochastic mapping $p(y \mid x)$.

At each channel use, the transmitter both harvests energy and transmits a symbol. The order of harvesting and transmission in a channel use is as follows: $S_{i}$ denotes the energy available in the battery at channel use $i$. The transmitter observes the available battery energy $S_{i}$ and transmits a symbol $X_{i}$. The energy of this symbol is constrained by the battery energy: $X_{i} \leq S_{i}$. After sending the symbol, the transmitter harvests energy. Energy arrivals (harvesting) is modeled as an i.i.d. process with $E_{i} \in\{0,1, \ldots,|\mathcal{E}|\}$ and $\operatorname{Pr}\left[E_{i}=e\right]=q_{e}$ for $e \in\{0,1, \ldots,|\mathcal{E}|\}$. Incoming energy $E_{i}$ is first stored in the battery, if there is space, before it is used for transmission. Since the battery has a finite size, energies may overflow and get wasted. The battery state is updated as:

$$
S_{i+1}=\min \left\{S_{i}-X_{i}+E_{i}, E_{\max }\right\}
$$

Initial battery state is $S_{1}=E_{\max }$ as it is always possible to fill up the battery and start communication without losing from the rate.

The energy arrival $E_{i}$ is available at the receiver. In view of (1) and the physical channel model, the energy arrival $E_{i}$ and the channel output $Y_{i}$ evolve according to the following joint distribution:

$$
p\left(e_{i}, y_{i} \mid x^{i}, e^{i-1}\right)=p\left(e_{i}\right) p\left(y_{i} \mid x_{i}\right), \quad x_{i} \leq s_{i}
$$

where $s_{i}$ is the battery energy level at the $i$ th channel use. We note that the product form $p\left(y_{i} \mid x_{i}\right) p\left(e_{i}\right)$ in (2) suggests that the channel and the energy arrivals are independent; however, due to the constraint $x_{i} \leq s_{i}$, there is a time correlation in the transmitted input sequence, i.e., the battery imposes memory constraint in the channel input sequence.

\section{MAIN RESUlt}

We state the main result of this paper in the following theorem:

Theorem 1 The capacity of the energy harvesting channel with energy arrival side information at the receiver in addition to the transmitter is:

$$
C=\lim _{n \rightarrow \infty} \max _{p\left(x_{i} \mid s_{i}\right)} \frac{1}{n} I\left(X^{n} ; Y^{n} \mid E^{n}\right)
$$

Moreover, the capacity is invariant to the availability of noncausal knowledge of energy arrivals.

Before proving Theorem 1, we provide the following corollary:
Corollary 1 The following rate $R$ is achievable with energy arrival side information at both sides:

$$
R=\lim _{n \rightarrow \infty} \max _{p\left(x_{i} \mid s_{i}\right)} \frac{1}{n} \sum_{i=1}^{n} I\left(X_{i} ; Y_{i} \mid S_{i}\right)
$$

We, next, comment on the capacity expression in (3) and achievable rate in (4). Note that the capacity achieving input sequence is obtained by using an input distribution at channel use $i, p\left(x_{i} \mid s_{i}\right)$, that depends only on the current battery level $s_{i}$. In fact, this could be viewed as an extension of [13, Theorem 3] where current state information is sufficient for encoding; however, realization of the whole energy arrival sequence is needed for decoding. Battery state information is inherently available at the transmitter; therefore, this is a feasible encoding scheme. However, note that the battery state information is not available at the receiver side. That is, even when the battery state information is not available at the receiver, the rate $R$ in (4) and possibly higher rates are achievable. The conditioning on the battery state $S_{i}$ in the mutual information in (4) should not be interpreted as the battery state information being available at the receiver. In fact, when the battery state information is available at the receiver, the capacity is found as in [4].

\section{Proofs of Theorem 1 AND Corollary 1}

\section{A. Proof of Theorem 1}

We start the proof with the converse part and assume that the energy arrival sequence $E^{n}$ is available at both the transmitter and the receiver non-causally. Note that non-causal knowledge of energy arrivals is stronger than the original system assumptions, yielding an upper bound for the rate achievable under them. First, define $\mathcal{F}\left(E^{n}\right)$ as the support set of the $X^{n}$ sequence lies in a constrained set defined by the battery dynamics:

$$
\begin{aligned}
\mathcal{F}_{n}\left(E^{n}\right) \triangleq\left\{p\left(x^{n}\right)\right. & \text { with supoort set } x_{i} \leq s_{i}, \\
& s_{i+1}=\min \left\{s_{i}-x_{i}+E_{i}, E_{\max }\right\}, \\
& \left.s_{1}=E_{\max }\right\}
\end{aligned}
$$

The code is generated based on the non-causal knowledge of the energy arrivals: For any given $E^{n}$ sequence, the codewords are constrained to lie in the set $\mathcal{F}_{n}\left(E^{n}\right)$. Note that this is equivalent to causal conditioning for the code symbol energy at each channel use. We have the following inequalities:

$$
\begin{aligned}
n R-H\left(W \mid Y^{n}, E^{n}\right) & =I\left(W ; Y^{n}, E^{n}\right) \\
& \leq I\left(X^{n} ; Y^{n} \mid E^{n}\right) \\
& \leq \sup _{p\left(x^{n}\right) \in \mathcal{F}_{n}\left(E^{n}\right)} I\left(X^{n} ; Y^{n} \mid E^{n}\right)
\end{aligned}
$$

where (7) follows from the data processing inequality and the fact that message $W$ is independent of the energy arrivals $E^{n}$. Taking the limit as $n$ tends to infinity and using Fano's inequality, we reach the following inequality:

$$
R \leq \lim \inf _{n \rightarrow \infty} \frac{1}{n} \sup _{p\left(x^{n}\right) \in \mathcal{F}\left(E^{n}\right)} I\left(X^{n} ; Y^{n} \mid E^{n}\right)
$$


Now, define $C_{n}=\sup _{p\left(x^{n}\right) \in \mathcal{F}\left(E^{n}\right)} I\left(X^{n} ; Y^{n} \mid E^{n}\right)$. We next show that $C_{n}$ is a sub-additive sequence. Note the following relation:

$$
\begin{aligned}
& \mathcal{F}_{n+m}\left(E^{n+m}\right) \\
& \quad \subseteq\left\{p\left(x^{n+m}\right): p\left(x_{1}^{n}\right) \in \mathcal{F}_{n}\left(E^{n}\right), p\left(x_{n+1}^{n+m}\right) \in \mathcal{F}_{m}\left(E_{n+1}^{n+m}\right)\right\}
\end{aligned}
$$

where (10) follows from the fact that in the definition of $\mathcal{F}_{n}\left(E^{n}\right)$ in (5), initial battery energy is $E_{\text {max }}$. Define the set on the right hand side of $(10)$ as $\tilde{\mathcal{F}}\left(E^{n+m}\right)$. We reach the following inequalities:

$$
\begin{aligned}
C_{n+m} & =\sup _{p\left(x^{n+m}\right) \in \mathcal{F}\left(E^{n+m}\right)} I\left(X^{n+m} ; Y^{n+m} \mid E^{n+m}\right) \\
\leq & \sup _{p\left(x^{n+m}\right) \in \tilde{\mathcal{F}}\left(E^{n+m}\right)} I\left(X^{n+m} ; Y^{n+m} \mid E^{n+m}\right) \\
\leq & \sup _{p\left(x^{n}\right) \in \mathcal{F}\left(E^{n}\right)} I\left(X^{n} ; Y^{n} \mid E^{n}\right) \\
& \quad+\sup _{p\left(x^{m}\right) \in \mathcal{F}\left(E_{n+1}^{n+m}\right)} I\left(X^{m} ; Y^{m} \mid E_{n+1}^{n+m}\right) \\
& =C_{n}+C_{m}
\end{aligned}
$$

where (12) follows from the relation in (10) and (13) is due to the fact that $p\left(x^{n}\right)$ and $p\left(x_{n+1}^{n+m}\right)$ could be independently selected in $\tilde{\mathcal{F}}\left(E^{n+m}\right)$. Finally, (14) follows from the fact that the $E_{i}$ sequence is i.i.d. and hence $C_{m}=$ $\sup _{p\left(x^{m}\right) \in \mathcal{F}\left(E_{n+1}^{n+m}\right)} I\left(X^{m} ; Y^{m} \mid E_{n+1}^{n+m}\right)$ is independent of the time index $n$. By Fekete's lemma, we have

$$
\lim \inf _{n \rightarrow \infty} \frac{1}{n} C_{n}=\inf _{n} \frac{C_{n}}{n}=\lim _{n \rightarrow \infty} \frac{1}{n} C_{n}
$$

We now show that the rate $R=\lim _{n \rightarrow \infty} \frac{C_{n}}{n}$ is achievable with non-causal knowledge of the energy arrivals. Fix $n$ and consider all possible $E^{n}=e^{n}$ sequences. Find $\sup _{p\left(x^{n}\right) \in \mathcal{F}_{n}\left(e^{n}\right)} I\left(X^{n} ; Y^{n} \mid e^{n}\right)$ for all $e^{n}$. Then, we perform the encoding over blocks of $n$ channel uses and insert zero symbols $o(n)$ channel uses so that the battery returns to the full energy state. That is, each block is of length $n+o(n)$ and consider $k$ such blocks: the $i$ th block consists of $n$ code symbols generated from the distribution that achieves $\sup _{p\left(x^{n}\right) \in \mathcal{F}_{n}\left(e_{(i-1)(n+o(n))+1}^{(i-1)(n+o(n))+n}\right)} I\left(X^{n} ; Y^{n}\right)$ and they are followed by $o(n)$ zero symbols. Since $e_{(i-1)(n+o(n))+1}^{(i-1)(n+o(n))+n}$ are independent for all $i$, we conclude that as the number of blocks $k$ grows to infinity, by multiplexing over different codebooks as in [14], the rate $\sup _{p\left(x^{n}\right) \in \mathcal{F}_{n}\left(E^{n}\right)} I\left(X^{n} ; Y^{n} \mid E^{n}\right)$ is achieved provided that the initial full battery state is guaranteed at the beginning of each block. However, by selecting $o(n)$ such that $o(n) \rightarrow \infty$ as $n \rightarrow \infty$, (e.g., $o(n)=\log (n))$, we conclude that

$$
\lim _{n \rightarrow \infty} \frac{C_{n}}{n+o(n)}
$$

is indeed achievable. This proves that $\lim _{n \rightarrow \infty} \frac{C_{n}}{n}$ is achievable. Note that [9] uses a similar achievable scheme when the energy arrivals are deterministic. However, the waiting time is finite in that case as the energy arrivals are deterministic and battery is finite.
We have just shown that $\lim _{n \rightarrow \infty} \frac{C_{n}}{n}$ is the capacity with non-causal knowledge of energy arrivals. To complete the proof, we prove that in the above achievable scheme, only causal knowledge of the energy arrivals is sufficient. In other words, we prove the following equality:

$$
\sup _{\left.x^{n}\right) \in \mathcal{F}\left(E^{n}\right)} I\left(X^{n} ; Y^{n} \mid E^{n}\right)=\sup _{p\left(x_{i} \mid s_{i}\right)} I\left(X^{n} ; Y^{n} \mid E^{n}\right)
$$

To this end, we express the objective as:

$$
\begin{aligned}
I\left(X^{n}\right. & \left.; Y^{n} \mid E^{n}\right) \\
& =H\left(Y^{n} \mid E^{n}\right)-H\left(Y^{n} \mid X^{n}, E^{n}\right) \\
& =\sum_{i=1}^{n} H\left(Y_{i} \mid Y^{i-1}, E^{n}\right)-H\left(Y_{i} \mid Y^{i-1}, X^{n}, E^{n}\right) \\
& \leq \sum_{i=1}^{n} H\left(Y_{i} \mid Y^{i-1}, E^{i-1}\right)-H\left(Y_{i} \mid X^{i}, E^{i-1}\right) \\
& =\sum_{i=1}^{n} H\left(Y_{i} \mid Y^{i-1}, E^{i-1}\right)-H\left(Y_{i} \mid X_{i}, S_{i}\right)
\end{aligned}
$$

where (20) follows from conditioning reduces entropy and the fact that channel is DMC, i.e., $p\left(y_{i} \mid x^{i}, y^{i-1}\right)=p\left(y_{i} \mid x_{i}\right)$ with $x_{i} \leq s_{i}=f\left(x^{i-1}, e^{i-1}\right)$; (21) also follows from the fact that $s_{i}=f\left(x^{i-1}, e^{i-1}\right)$, a deterministic function. Next, we show that it suffices to consider input distributions in the form of $p\left(x_{i} \mid s_{i}\right)$ to maximize $(21)$.

Let us fix $p\left(x_{i} \mid x^{i-1}, e^{i-1}\right)$ for $i=1, \ldots, n-1$ and maximize the objective over $p\left(x_{n} \mid x^{n-1}, e^{n-1}\right)$. Note that fixing $p\left(x_{i} \mid x^{i-1}, e^{i-1}\right)$ for $i=1, \ldots, n-1$ fixes $H\left(Y_{i} \mid Y^{i-1}, E^{i-1}\right)$ and $H\left(Y_{i} \mid X_{i}, S_{i}\right)$ for $i=1, \ldots, n-1$ and $p\left(s_{i}\right)$ for $i=1, \ldots, n$. The remaining term, $H\left(Y_{n} \mid Y^{n-1}, E^{n-1}\right)-$ $H\left(Y_{n} \mid X_{n}, S_{n}\right)$, is a function of $p\left(x_{n} \mid x^{n-1}, e^{n-1}\right)$. In particular, $H\left(Y_{n} \mid X_{n}, S_{n}\right)$ is just a function of $p\left(x_{n} \mid s_{n}\right)$ when $p\left(s_{n}\right)$ is fixed. Hence, it suffices to show that $H\left(Y_{n} \mid Y^{n-1}, E^{n-1}\right)$ is maximized by distributions of the form $p\left(x_{i} \mid s_{i}\right)$. To this end, we note that for any given $p\left(x^{n-1} \mid e^{n-1}\right), Y^{n-1}=y^{n-1}$ and $E^{n-1}=e^{n-1}$, a distribution is generated on $x^{n-1}$, denoted as $p\left(x^{n-1} \mid y^{n-1}, e^{n-1}\right)$, with the support set $\mathcal{F}_{n-1}\left(e^{n-1}\right)$. We have:

$$
\begin{gathered}
p\left(y_{n} \mid y^{n-1}, e^{n-1}\right)=\sum_{x^{n}, s_{n}} p\left(y_{n} \mid x_{n}\right) p\left(x_{n} \mid s_{n}, x^{n-1}, e^{n-1}\right) \\
p\left(x^{n-1} \mid y^{n-1}, e^{n-1}\right)
\end{gathered}
$$

In addition, the next battery energy level distribution is:

$$
\begin{gathered}
p\left(s_{n+1}\right)=\sum_{x^{n}, e^{n}} p\left(s_{n+1} \mid x_{n}, s_{n}, e_{n}\right) p\left(x_{n} \mid s_{n}, x^{n-1}, e^{n-1}\right) \\
p\left(x^{n-1}, e^{n}\right)
\end{gathered}
$$

where $p\left(s_{n+1} \mid x_{n}, s_{n}, e_{n}\right)=1$ if and only if $s_{n+1}=\min \left\{s_{n}-\right.$ $\left.x_{n}+e_{n}, E_{\max }\right\}$ and 0 otherwise.

We select $p\left(x_{n} \mid s_{n}, x^{n-1}, e^{n-1}\right)$ as in the following

$$
\begin{aligned}
& \hat{p}\left(x_{n} \mid s_{n}, x^{n-1}, e^{n-1}\right)=p\left(x_{n} \mid s_{n}\right)= \\
& \sum_{x^{n-1}, e^{n-1}} p\left(x_{n} \mid s_{n}, x^{n-1}, e^{n-1}\right) p\left(x^{n-1}, e^{n-1}\right)
\end{aligned}
$$


As $H\left(Y_{n} \mid Y^{n-1}, E^{n-1}\right)$ is a concave function of $p\left(y_{n} \mid y^{n-1}, e^{n-1}\right)$, we deduce from (22) and by Jensen's inequality that it yields higher $H\left(Y_{n} \mid Y^{n-1}, E^{n-1}\right)$ value. Moreover, this selection $\hat{p}\left(x_{n} \mid s_{n}, x^{n-1}, e^{n-1}\right)$ does not change the remaining energy distribution $p\left(s_{n+1}\right)$ in view of (23). In particular, $p\left(x^{n-1}, y^{n-1}, e^{n}\right)=p\left(e_{n}\right) p\left(x^{n-1}, y^{n-1}, e^{n-1}\right)$ Since this is true for any $n$, we prove that $p\left(x_{i} \mid s_{i}\right)$ is sufficient for the optimization problem on the left hand side of (17).

To conclude, we have shown that even under non-causal knowledge of $E^{n}$, the rate $R=\inf _{n} \frac{C_{n}}{n}$ is the highest achievable rate and it can be achieved by an encoding scheme that determines the channel input $x_{i}$ as a stochastic function of only the battery state $s_{i}$. This result and its proof could be viewed as an extension of the coding theorem in [14].

We remark that the energy harvesting channel with deterministic energy arrivals and no side information considered in [9] is a special case of the problem we address in the current paper. In view of Theorem 1, encoding based on the current battery state $s_{i}$ is sufficient for achieving the capacity when energy arrivals are deterministic.

\section{B. Proof of Corollary 1}

In order to prove Corollary 1 , it suffices to prove the following inequality:

$$
\sup _{p\left(x_{i} \mid s_{i}\right)} I\left(X^{n} ; Y^{n} \mid E^{n}\right) \geq \sup _{p\left(x_{i} \mid s_{i}\right)} \sum_{i=1}^{n} I\left(X_{i} ; Y_{i} \mid S_{i}\right)
$$

We first observe from (21) that whenever $p\left(x_{i} \mid x^{i-1}, e^{i-1}\right)=$ $p\left(x_{i} \mid s_{i}\right)$ :

$$
I\left(X^{n} ; Y^{n} \mid E^{n}\right)=\sum_{i=1}^{n} H\left(Y_{i} \mid Y^{i-1}, E^{i-1}\right)-H\left(Y_{i} \mid X_{i}, S_{i}\right)
$$

Hence, it suffices to prove:

$$
\begin{gathered}
\sup _{p\left(x_{i} \mid s_{i}\right)} \sum_{i=1}^{n} H\left(Y_{i} \mid Y^{i-1}, E^{i-1}\right)-H\left(Y_{i} \mid X_{i}, S_{i}\right) \\
\geq \sup _{p\left(x_{i} \mid s_{i}\right)} \sum_{i=1}^{n} I\left(X_{i} ; Y_{i} \mid S_{i}\right)
\end{gathered}
$$

We note that since $X_{i}=f_{i}\left(S_{i}\right)$, the following Markov chain holds:

$$
Y^{i-1}, E^{i-1} \leftrightarrow S_{i} \leftrightarrow X_{i} \leftrightarrow Y_{i}
$$

Then, we have the following inequality due to the data processing inequality:

$$
H\left(Y_{i} \mid Y^{i-1}, E^{i-1}\right) \geq H\left(Y_{i} \mid S_{i}\right)
$$

This proves the desired result in (27).

\section{Solution of (4) Via Dynamic Programming}

The optimization problem in (4) can be solved by dynamic programming for fixed $n$. Assume $s_{n}$ is fixed and calculate the value function for all $s_{n} \in\left\{0, \ldots, E_{\max }\right\}$ as follows:

$$
J_{n}\left(s_{n}\right)=\max _{p\left(x_{n} \mid s_{n}\right)} I\left(X_{n} ; Y_{n} \mid S_{n}=s_{n}\right)
$$

Then, calculate the value function for $i=1, \ldots, n-1$ :

$J_{i}\left(s_{i}\right)=\max _{p\left(x_{i} \mid s_{i}\right)} I\left(X_{i} ; Y_{i} \mid S_{i}=s_{i}\right)+\sum_{s_{i+1}=0}^{E_{\max }} p\left(s_{i+1} \mid s_{i}\right) J_{i}\left(s_{i+1}\right)$

Note that $p\left(s_{i+1} \mid s_{i}\right)$ is calculated as:

$$
p\left(s_{i+1} \mid s_{i}\right)=\sum_{e_{i}, x_{i}, s_{i+1}} p\left(s_{i+1} \mid s_{i}, x_{i}, e_{i}\right) p\left(e_{i}\right) p\left(x_{i} \mid s_{i}\right)
$$

Since $s_{i+1}=\min \left\{\left(s_{i}-x_{i}+e_{i}\right)^{+}, E_{\max }\right\}$ is a deterministic function, $p\left(s_{i+1} \mid s_{i}, x_{i}, e_{i}\right)$ is just an indicator function.

\section{The Channel with Output Feedback}

In this section, we consider the capacity of the channel under study when the channel output feedback is also present at the transmitter. It is well-known that in non-anticipative systems feedback does not increase capacity [15]. We will establish this result for our particular energy harvesting channel.

We first note that the $n$-letter mutual information in (3) is the maximum directed information from the input $X^{n}$ to the output $Y^{n}$ given the energy arrivals $E^{n}$ :

$$
\begin{aligned}
I\left(X^{n} ; Y^{n} \mid E^{n}\right)= & \sum_{i=1}^{n} I\left(X^{n} ; Y_{i} \mid Y^{i-1}, E^{n}\right) \\
= & \sum_{i=1}^{n} I\left(X^{i} ; Y_{i} \mid Y^{i-1}, E^{n}\right) \\
& \quad+I\left(X_{i+1}^{n} ; Y_{i} \mid X^{i}, Y^{i-1}, E^{n}\right) \\
= & \sum_{i=1}^{n} I\left(X^{i} ; Y_{i} \mid Y^{i-1}, E^{n}\right) \\
= & I\left(X^{n} \rightarrow Y^{n} \mid E^{n}\right)
\end{aligned}
$$

where (35) is due to the fact that the Markov chain $Y_{i} \leftrightarrow$ $\left(X^{i}, Y^{(i-1)}, E^{n}\right) \leftrightarrow X_{i+1}^{n}$ holds for all $i$. This Markov chain holds in view of the fact that $X_{i+1}^{n}$ is determined as a function of message $W, X^{i}$ and $E^{i}$. Therefore, $Y_{i}$ is independent of $X_{i+1}^{n}$ given $X^{i}, Y^{(i-1)}, E^{n}$. This renders the term $I\left(X_{i+1}^{n} ; Y_{i} \mid X^{i}, Y^{i-1}, E^{n}\right)=0$. See also [16, Proposition 4.2.2].

Next, we observe that $X^{i}$ is independent of $E_{i}$ in view of the fact that $E_{i}$ is an i.i.d. sequence and the constraint set for $X^{i}$ is determined by $E^{i-1}$. Therefore, we have the following:

$$
\begin{aligned}
I\left(X^{n} ; Y^{n} \mid E^{n}\right) & =\sum_{i=1}^{n} I\left(X^{i} ; Y_{i} \mid Y^{i-1}, E^{n}\right) \\
& =\sum_{i=1}^{n} I\left(X^{i} ; Y_{i} \mid Y^{i-1}, E^{i-1}\right) \\
& =\sum_{i=1}^{n} I\left(X^{i} ; Y_{i}, E_{i} \mid Y^{i-1}, E^{i-1}\right) \\
& =I\left(X^{n} \rightarrow Y^{n}, E^{n}\right)
\end{aligned}
$$




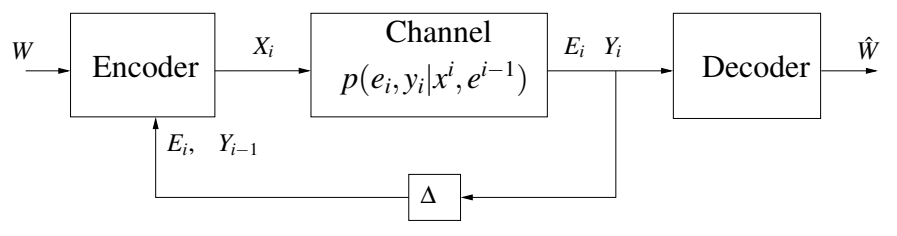

Fig. 2. Virtual channel model with feedback. Presence of the channel output feedback $Y$ does not affect the capacity.

Since $\lim _{n \rightarrow \infty} \frac{1}{n} \sup _{p\left(x^{n}\right) \in \mathcal{F}\left(E^{n}\right)} I\left(X^{n} ; Y^{n} \mid E^{n}\right)$ exists, we conclude that $\lim _{n \rightarrow \infty} \frac{1}{n} \sup _{p\left(x^{n}\right) \in \mathcal{F}\left(E^{n}\right)} I\left(X^{n} \rightarrow Y^{n}, E^{n}\right)$ also exists. In other words, the directed mutual information spectrum of the channel in (2) consists of a single point only. Note that the channel in (2) falls into the most general category of channels with feedback in [16]. In view of the general capacity formula for channels with feedback in [16, Theorem 4.4.1] and the fact that $\lim _{n \rightarrow \infty} \frac{1}{n} I\left(X^{n} \rightarrow Y^{n}, E^{n}\right)$ exists, we conclude that this limit is the capacity of the channel in (2) with feedback.

Theorem 2 When energy arrival side information is causally available at the transmitter and the receiver in the discrete memoryless energy harvesting channel, the channel output feedback does not increase the capacity.

We finally remark that in the case of an infinite-sized battery, the capacity is not affected by the presence of energy arrival side information at the receiver side, see also [1, Section IV]. Moreover, in view of Theorem 2, the presence of channel output feedback does not affect the capacity either.

\section{NUMERICAL RESULTS}

In this section, we evaluate the capacity bounds $C_{n}$ and achievable rates with and without receiver side information. We consider a binary symmetric channel with crossover probability $p_{e}$. We select $E_{\max }=1$ and i.i.d. energy arrivals with $P\left[E_{i}=1\right]=0.5$. In Fig. 3, we plot the achievable rates with and without receiver side information. We also include plots of the capacity for $E_{\max }=\infty$. The achievable rate with energy side information at the transmitter only is calculated by using the method reported in [5], [7]. Moreover, we plot capacities with battery state information at the receiver using [4]. Note that capacity with energy side information and battery side information match when channel is noiseless. Moreover, we observe that $C_{n}$ for $n=7$ yields a tighter bound as it lies below the capacity with battery state information at the receiver for most $p_{e}$ values.

\section{CONCLUSION}

We determined the capacity of an energy harvesting channel with an energy harvesting transmitter and energy arrival side information available at the transmitter and receiver sides. We first found an $n$-letter capacity expression and showed that the optimal coding is based on only current battery state $s_{i}$. Next, we showed that the capacity is expressed as maximum directed information between the input and the output. Moreover, we proved that the channel output feedback does not increase the capacity.

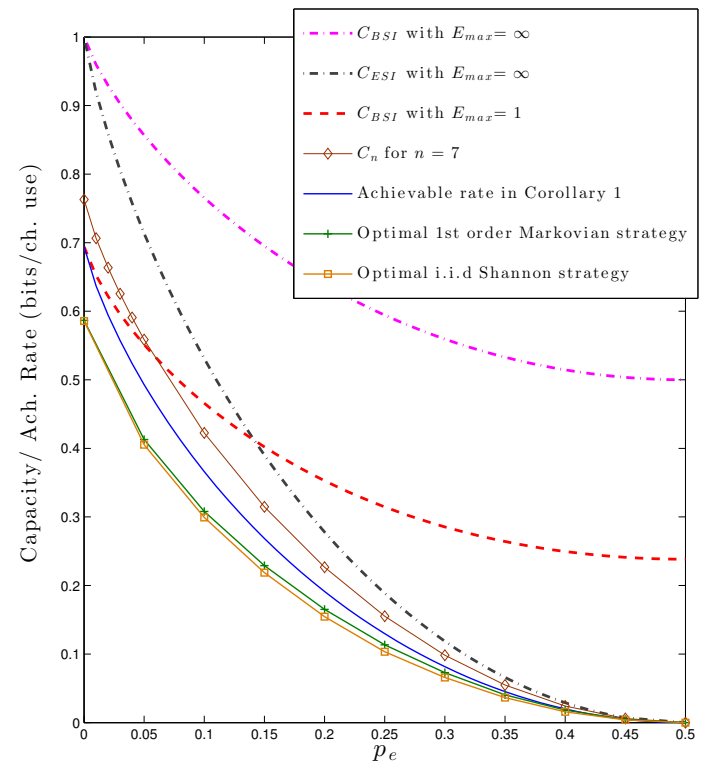

Fig. 3. The capacities with energy arrival and battery side information at the receiver side and achievable rates with side information only at the transmitter in a $\operatorname{BSC}\left(p_{e}\right)$. The plot is with respect to the channel crossover probability $p_{e}$ for $P\left[E_{i}=0\right]=0.5=P\left[E_{i}=1\right]$.

\section{REFERENCES}

[1] O. Ozel and S. Ulukus. Achieving AWGN capacity under stochastic energy harvesting. IEEE Trans. on Inform. Theory, 58(10):6471-6483, Oct. 2012.

[2] O. Ozel and S. Ulukus. AWGN channel under time-varying amplitude constraints with causal information at the transmitter. In Asilomar Conf., Nov. 2011.

[3] C. E. Shannon. Channels with side information at the transmitter. IBM journal of Research and Development, 2(4):289-293, 1958.

[4] O. Ozel, K. Tutuncuoglu, S. Ulukus, and A. Yener. Capacity of the discrete memoryless energy harvesting channel with side information. In IEEE ISIT, June 2014.

[5] K. Tutuncuoglu, O. Ozel, A. Yener, and S. Ulukus. Binary energy harvesting channel with finite energy storage. In IEEE ISIT, July 2013

[6] K. Tutuncuoglu, O. Ozel, A. Yener, and S. Ulukus. Improved capacity bounds for the binary energy harvesting channel. In IEEE ISIT, June 2014.

[7] W. Mao and B. Hassibi. On the capacity of a communication system with energy harvesting and a limited battery. In IEEE ISIT, July 2013.

[8] Y. Dong and A. Ozgur. Approximate capacity of energy harvesting communication with finite battery. In IEEE ISIT, June 2014.

[9] V. Jog and V. Anantharam. An energy harvesting AWGN channel with a finite battery. In IEEE ISIT, June 2014.

[10] J. Yang and S. Ulukus. Optimal packet scheduling in an energy harvesting communication system. IEEE Trans. Comm., 60(1):220-230, January 2012.

[11] K. Tutuncuoglu and A. Yener. Optimum transmission policies for battery limited energy harvesting nodes. IEEE Trans. Wireless Comm., 11(3):1180-1189, March 2012.

[12] O. Ozel, K. Tutuncuoglu, J. Yang, S. Ulukus, and A. Yener. Transmission with energy harvesting nodes in fading wireless channels: Optimal policies. IEEE Jour. on Selected Areas in Commun., 29(8):1732-1743, September 2011.

[13] G. Caire and S. Shamai. On the capacity of some channels with channel state information. IEEE Trans. on Information Theory, 45:2007-2019, September 1999.

[14] A. Goldsmith and P. Varaiya. Capacity of fading channels with channel side information. IEEE Trans. on Inform. Theory, 43(6):1986-1992, November 1997.

[15] J. Massey. Causality, feedback and directed mutual information. In IEEE ISITA, Honolulu, HI, Nov. 1990.

[16] S. Tatikonda. Control under communication constraints. PhD Thesis, MIT, Cambridge, MA, September 2000. 